\title{
Macedonian Medical Doctor of Philosophy (PhD) Theses Defended in 2012
}

\author{
Macedonian Journal of Medical Sciences \\ Institute of Immunobiology and Human Genetics, Faculty of Medicine, Ss. Cyril and Methodius University in Skopje, Skopje, \\ Republic of Macedonia
}

\begin{abstract}
Citation: Macedonian Journal of Medical Sciences. Macedonian Medical Doctor of Philosophy (PhD) Theses Defended in 2012 . Philosophy (PhD) Theses Defended in 2012. Maced J Med Sci. 2013 Mar 15; $6(1) .106-123$. Key words: Doctor of Philosophy (PhD); Medical research; Republic of Macedonia.

"Correspondence: Macedonian Journal of Medical Sciences. Institute of Immunobiology and Human Genetics, Faculty of Medicine, Ss. Cyril and Methodius University in Skopje, Republic of Macedonia. 50 Divizija No 16, PO Box 60, 1109 Skopje, Republic of Macedonia. Telephone: +3892 3110556. Telefax: +389 2 3110558. EMail: mjms@ukim.edu.mk

Received: 22-Feb-2013; Revised: 28-Feb2013; Accepted: 02-Mar-2013; Online first: 04-Mar-2013

Copyright: (c) 2013 Macedonian Journal of Medical Sciences. This is an open-access article distributed under the terms of the Creative Commons Attribution License, which permits unrestricted use, distribution, and reproduction in any medium, provided the original author and source are credited.

Competing Interests: The author have declared that no competing interests exist.
\end{abstract}

\begin{abstract}
We present English abstracts of $\mathrm{PhD}$ theses defended in 2012 at the Faculty of Medicine, Ss. Cyril and Methodius University in Skopje, Republic of Macedonia. English summaries are published as they are translated by authors and included in the final version of defended PhD. Macedonian Medical Doctor of Philosophy (PhD) theses are deposited in the Central Medical Library and National and University Library "St. Kliment Ohridski" in Skopje.

At the Faculty of Medicine in Skopje $21 \mathrm{PhD}$ theses there were defended in 2012, four of them without English abstract (19.0\%): two from the University Clinic of Digestive Surgery, one from the University Children's Hospital, and one from University Clinic of Rheumatology. Eleven PhDs are without Key words $(52.4 \%)$, and eight of them are with structured abstracts $(38.1 \%)$. One $\mathrm{PhD}$ is even without mentor (University Clinic for Orthopaedic Surgery).

Editorial Board does not take any responsibility either for the content, nor the quality of the abstracts. Primary responsibility for the quality of the $\mathrm{PhD}$ theses belongs to the mentors, to the institutions they are representing, and to the Vice-Dean of science. They should be more actively involved in the preparation of Doctor of Philosophy theses in order international standards to be achieved.
\end{abstract}

Defended PhDs can be cited as they are published in this and in the previous reports.

\section{Sadije Namani. Factors which favourite neurological complications of bacterial meningitis in children [PhD Thesis]. Skopje, Republic of Macedonia: University Clinic of Infectious Diseases, Faculty of Medicine, Ss Cyril and Methodius University of Skopje; 2012. Maced J Med Sci. Maced J Med Sci. 2013 Mar 15; 6(1):106- 123.}

Despite the progress made in medicine, bacterial meningitis remains one of ten most common infections causes of deaths worldwide and up to onehalf of survivors have significant neurologic sequelae.
The risk of developing complications has been shown to be related to the age and underlying health status of the patient, the causative pathogen, the severity and duration of illness at the time of presentation, and to delays in the initiation of antibiotic therapy.

The aim of this dissertation is to contribute in identification of factors that influence the neurological complications of bacterial meningitis in children.

The dissertation is consisted of three parts. In the first part 17 relevant predictors and their association with the incidence of neurological complications in children 
categorized into specific age groups (0-1 years old, $>1-6$ years of age and $>6-16$ years of age) were studied. The chosen predictors were: gender, comorbidity, duration of illness before being diagnosed for bacterial meningitis, previous treatment with antibiotics, presentation of seizures, the presence of altered mental status at the time of presentation, the presence of neurologic deficits at the time of admission, the presence/absence of a primary infectious focus, the causative pathogens and their resistance to antibiotics, the initial antimicrobial therapy, dexamethasone use, whether the patient had a community- or hospital-acquired infection and cerebrospinal fluid (CSF) laboratory parameters: the initial pleocytosis in CSF, CSF/blood glucose ratio, increased protein levels in CSF and pleocytosis in CSF after 48 hours.

In the second part the same predictors were analyzed for their association with the incidence of neurologic complications in adults categorized into specific age groups (>16-26 years of age, $>26-60$ years of age and $>60$ years of age).

In the third part the date comparison is made between children and adults concerning predictors for neurologic complications as well as the incidence of neurologic complications in both group-ages.

In this study 123 patients treated for bacterial meningitis were analyzed; 77 patients were children at age of $0-16$ years $(62.60 \%)$, and 46 were adults older than $16(37.39 \%)$ with ratio $1.67: 1$.

Out of 77 children, 33 cases manifested acute neurologic complications (42.86\%) while two patients died with the overall mortality rate of $2.59 \%$.

Out of 46 adult patients treated for bacterial meningitis, 17 patients manifested acute neurologic complications $(36.95 \%)$, while 6 patients died $(\mathrm{M}=13.04 \%)$.

Children under 12 months of age for 4.09 times had higher risk for development of neurologic complications $\mathrm{OR}=4.09(1.35<0 . \mathrm{R}<12.43)$ compared to children from $>1-6$ and for 22.5 times had higher risk for developing neurologic complications compared to children >6-16 (OR=22.5 (4.21<O.R<120.15).

Age under 12 months, presence of seizures prior to being diagnosed for bacterial meningitis, altered mental status at admission, presence of neurologic deficit, the initial antimicrobial therapy with 2 antibiotics, the use of dexamethasone and elevated proteins in CSF were the factors associated to the increased risk for development of neurologic complications in children with statistical significance.

Female patients and illness duration $>48$ hours prior being diagnosed for bacterial meningitis were factors associated with increased risk for development of neurologic complications in children but without statistical significance.
Altered mental state, the initial antimicrobial therapy with 2 antibiotics, resistance of causative pathogens to antibiotics, the use of dexamethasone and pleocytosis > $5000 / \mathrm{mm}^{3}$ after 48 hours were more frequently presented in the group at age of $0-1$ $(p<0.05)$.

For the adults no statistical differences in the occurrence of neurologic complications were identified concerning group ages $(p=0.41)$.

The previous treatment with antibiotics, altered mental state at admission, especially coma, presence of neurologic deficit at admission and isolated etiologic agent were the factors associated with increased risk for development of neurologic complications in adults with statistical significance.

Male patients and illness duration $>48$ hours being diagnosed for bacterial meningitis were the factors associated with increased risk for development of neurologic complications in adults, but without statistical significance.

The highest incidence of bacterial meningitis and neurologic complications occurred in children under 12 months. Children had 1.28 times higher risk for development of neurologic complications comparing to adults $/ O R=1.28 /$, but without statistical significance.

The illness duration before being diagnosed for bacterial meningitis was longer with the adults compared to the age of the children groups $(p<0.001)$.

Comorbidity was more frequently presented with adults $(p<0.001)$.

The oral therapy was more frequently given to children, while the parenteral therapy was given to the adults.

Altered mental state at admission-somnolence was more frequent with children, while spoor and coma were more frequently presented with adults $(p<0.001)$.

CSF / blood glucose ratio $<0.20$ was more frequently presented in adults.

As a conclusion, children were exposed to a greater risk for developing neurological complications of bacterial meningitis especially young ages $<12$ months of age while adults $>60$ years of age had a greater risk for mortality from bacterial meningitis- the age extremes had the worst outcome.

Outcomes of this dissertation encourage additional research into the role of risk factors for developing neurologic complications as well a long follow up of children for determination of long term sequels such as hearing loss, seizures, neurologic deficits, neuropsychological impairment and developmental disabilities.

The study also recommends additional research for the relationship between intellectual, linguistic, learning, and reading skills with educational difficulty 
especially with children with a history of bacterial meningitis prior to 12 months of age being an important risk factor.

Implementation of vaccination, into national immunization programs in developing countries such as Kosovo, against three most common meningeal pathogens of bacterial meningitis in children (Neisseria meningitis, Streptococcus pneumonia and Haemophilus influenza) would be our future step in decreasing the incidence of bacterial meningitis in children.

Keywords: not available.

Defended: January 10, 2012.

Mentor: Prof. Dr. Zvonko Milenkovikj.

Nensi Manusheva. Spectral electroencephalographic differences in relation to clinical characteristics in patients with schizophrenia and bipolar disorder [PhD Thesis]. Skopje, Republic of Macedonia: University Psychiatry Clinic, Faculty of Medicine, Ss Cyril and Methodius University of Skopje; 2012. Maced J Med Sci. 2013 Mar 15; 6(1):106-123.

Introduction: In order to obtain neurophysiologic correlate for the disturbed behavior and thinking in psychiatric disorders variety of investigations was done focusing on quantitative electroencephalography (QEEG) parameters. The aim of this study was to investigate the differences with the expression of the diseases measured with clinical scales. Also, we tried to correlate the QEEG power spectrum to the clinical characteristics of the disease such as duration of the psychosis, duration of current episode, previous psychopharmacologic treatment and heredity.

Material and methods: Ninety subjects were enrolled in the study in the period between 2008-2010. We included 30 healthy subjects, $30 \mathrm{BD}$ patients $(46.66 \%$ with manic symptomatology and $53.33 \%$ with current depressive episode) and 30 schizophrenia patients (13 with positive symptomatology and 17 with negative symptoms). All patients fulfilled the ICD-10 diagnostic criteria for schizophrenia and $\mathrm{BD}$ and were treated at the University Psychiatry Clinic in Skopje. Complete medical history and clinical examination were performed in the evaluation of participants. For the evaluation of the severity of the disorder we used clinical scales: CGI (Clinical Global Impression scale); BPRS (Brief Psychiatric Rating Scale; PANSS (Positive and Negative Syndrome Scale); YMRS (Young Mania Rating Scale) and HAMD (Hamilton Depression Rating Scale). Neurophysiologic examination was performed using EBNeuro Galileo Mizar 25. QEEG recordings were performed between 9 a.m. - 1 p.m. while the subjects were awake with their eyes closed. The standard 10-20 electrodes placement system with 19 electrodes on the scalp and one on both earlobes were used in the recordings.
The resistance was kept below $10 \mathrm{k} \Omega$. The EEG digital recordings were screened visually and $25 \times 2$ second artifact-free epochs were selected from the background activity of the recording for subsequent analysis. As a result of the Fast Fourier Transformation, the averaged power spectral values of the delta $(0.5-3.5 \mathrm{~Hz})$, theta $(4.0-7.5 \mathrm{~Hz})$, alpha (8$12.5 \mathrm{~Hz})$, beta $1(13-19.5 \mathrm{~Hz})$ and beta $2(20.0-29.5$ $\mathrm{Hz})$ bands and also for alpha $1(8-9 \mathrm{~Hz})$ and alpha 2 (10-12 Hz) were produced for each of the electrodes separately. Afterwards we analyzed the spectral power in the different bands. In order to obtain topography of the main regions of interest the following electrode placements were chosen for analysis: F3; F4; C3; T3; T4; P3; P4; $\mathrm{O} 1$ and $\mathrm{O} 2$. Statistical analysis of the data was performed using the Statistical Package for the Social Sciences (SPSS) for Windows, version 13.0. We used mean and SD, percentages, the t-test for independent samples, Tukey HSD test, Yates' Chi square test and Mann Whitney non-parametric test. Analysis of the QEEG parameters was performed with ANOVA/MANOVA. We also use Spearman Rank Order Correlation.

Results: Statistical significant difference was obtained for the QEEG power spectra of alpha activity which was lower over all regions in the patients' groups. There were no differences in spectral power between the patients with $\mathrm{BD}$ and schizophrenia. Patients with schizophrenia were different from the healthy subjects and bipolar patients because they had decreased alpha 1 power spectrum over occipital regions. Schizophrenia patients with positive symptomatology compared to healthy subjects present differences in theta activity which was decreased over temporalcentral-parietal-occipital regions more pronounced on the left side. Also, alpha power spectrum activity was decreased over all leads such as alpha 1 and alpha 2 activities. Patients with negative schizophrenia syndrome compared to control healthy subjects were different over all leads because they have decreased alpha and alpha 2 power spectrum activities, and decreased spectral power of alpha 1 activity over frontal and parietal-occipital regions, but they have increased spectral power of beta 2 activity over frontal and temporal leads. There were no differences in spectral power between the patients with positive and the patients with negative syndrome of schizophrenia when they were compared with each other. Positive correlation was found in patients with schizophrenia for delta activity and PANSS total score and also for BPRS scores for thought disorder, depression and agitation/anxiety. Negative correlation was found for theta, alpha, alpha 1 and alpha 2 activity spectral power and BPRS score for agressivity and paranoid ideas. We found positive correlation for spectral alpha and alpha 2 power with the duration of the illness over frontal leads. Bipolar patients with current depressive episode compared to healthy subjects showed differences in decreased alpha and alpha 2 power spectrum over all leads, decreased beta 1 spectral 
power over central-parietal-occipital regions bilaterally and decreased beta2 activity over parietal leads. Bipolar patients with current manic symptomatology had decreased power spectrum of alpha and alpha 2 activity over frontal-temporal-parietal regions on the right hemisphere and over central-parietal-occipital regions on the left. Bipolar patients with actual depressive episode were different from the manic bipolar patients because they have more spectral power of the alpha activity over frontal regions. More alpha spectral power over occipital regions and central-parietal leads on the right, and also, larger spectral power of alpha 2 and beta 2 activities was present in bipolar manic patients when compared to depressed bipolar patients. Bipolar manic patients showed positive correlation of YMRS score and theta and delta activity over central leads bilaterally while negative correlation was present in depressive bipolar patients for HAMD score and delta activity over right frontal leads. In bipolar patients there were positive correlations between BPRS score for thought disorder and alpha and alpha 2 activity over frontal-temporal leads while delta, theta and beta 2 spectral power was positively correlated with BPRS score for agitation and aggressivity/paranoid ideas. In bipolar patients alpha and alpha 2 spectral power over left central-temporal regions was positively correlated with the duration of the disease. In both patient groups of schizophrenia and bipolar disorder no difference was found in the power spectrum activity in relation with the duration of the actual episode and the previous drug treatment. In bipolar patients there was a difference in alpha 2 power spectrum in relation to heredity.

Discussion: Our study confirmed that there are spectral QEEG differences in patients with schizophrenia and bipolar disorder which are disorganized in the power spectrum of basic EEG activity when compared to healthy control subjects. Correlations were found for the duration of the disease and power spectrum in the patients' group but there was no correlation with the duration of the current episode. This points out that these differences in the spectral power activity are reflection of the disease (trait-marker) and not a state marker. Correlations of the spectral EEG parameters with clinical scale scores were positive and showed us that larger the score of the psychopathology the basic EEG activity was more disorganized. These differences are reflections of the neurophysiologic aspects of the presented symptomatology.

Conclusion: Different spectral EEG parameters are correlated with some clinical characteristics of schizophrenia and bipolar disorder. Standardization of the methodology and knowledge of these QEEG parameters would allow wider application in the field of clinical psychiatry. In the future, these could help us in the distinction of the diseases in some peculiar cases and even in optimizing the treatment in a much more successful way.
Keywords: quantitative electroencephalography; power spectrum; schizophrenia; bipolar disorder; clinical scales.

Defended: January 20, 2012.

Mentor: Prof. Dr. Antoni Novotni.

Slavica Arsova Hadzi-Angelkovska. Quality of life in patients with schizophrenia [PhD Thesis]. Skopje, Republic of Macedonia: University Psychiatry Clinic, Faculty of Medicine, Ss Cyril and Methodius University of Skopje; 2012. Maced J Med Sci. 2013 Mar 15; 6(1):106-123.

The focus of this research is to analyze the quality of life in a group of subjects with schizophrenic disorder, observed through their personal and social functioning. The goals of this research are: with the help of structured clinical and several test steps to analyze the psychological functioning at the patients with schizophrenic disorder, treated with psycho socio-therapy steps in a environment of daily hospital conditions versus the ones who were ambulatory treated, furthermore to analyze the influence of familybased and social factors in their psychosocial functioning of the subjects with schizophrenia. Another goal is to analyze the relation and the meaning of negative and depressive symptomatology in the psychosocial functioning at the subjects with schizophrenic disorder, as well to observe the possible differences of that same subjects group regarding their: sex, age, education and the time when disorder is occurred for a first time.

Into the process of this research, 120 individuals divided in two equally separated groups of subjects with schizophrenia were included. The conducting group is constricted by 60 subjects from the both sexes which are having the schizophrenic disorder. The subjects from the both sexes are treated in the Daily Hospitals of the Psychiatry Clinic and Psychiatric hospital Skopje, Skopje. The subjects were diagnosed according to schizophrenia, they were over 18 years of age.

The control group is considered of 60 individuals from the both sexes who are having schizophrenic disorder. The group was formed by accidental choice from patients from the both sexes who are treated ambulatory in the previously above named hospitals. This control group was formed by the method of sex stratification due: sex, age and psych. Diagnose after the conducting group was formed.

The research was observed through structural test wise and clinical steps, with standardized psychiatric clinical interview for diagnosing and excluding other psychiatric disorders with Scale for clinical evaluation for the intensity of the schizophrenic symptology PANSS (The positive and negative syndrome scale) Kay et all. 1987 for the purpose of evaluation of the positive and negative schizophrenic symptology, 
Hamilton's scale for depression (HAMD) Hamilton, 1960 in the purpose of evaluating for the scale of intensity of the depressive simptomatology http://youtube.com/watch?feature=player_detailpage\& $v=k f f a c x f A 7 G 4$, it's used at already diagnosed patients. Furthermore scale for estimation of psychosocial functioning and quality of life at the subjects PSP (Personal and social performance scale) Morosini PI, Magliano L, Brambila L et all, 2000 , constructed of 4 parts (self care, socially useful activities, personal and social contacts and disturbing and aggressive acting). At all subjects not standardized questioner for socio demographic data was used, it was designated for the purpose of this research providing us with information regarding: age, sex, education and marriage status of our subjects, working status/relationship, the support from the family of the subject and the history of mental disorders into their families. The subjects from both groups were evaluated with previously above mentioned clinical tests/steps in the beginning of the research and in the end of the six months treatment period. For statistical analysis of the provided result, base was created in the software Statistica for Windows 7.0. As well for furthermore researching on the given data adequate statistical methodology was used. The final results from the research have shown that the majority of the subjects who are with schizophrenia are singles not married, high school level of education, unemployed with percentage of $68 \%$, with family amnesia for the presence of the mental disorder, only $39 \%$ from the subjects are describing their families as a supportive, and in the most of the cases the mental disorder was first acknowledge after the $18^{\text {th }}$ year of life. In this research female subjects were present with $46 \%$ and males with $54 \%$, the age average were 44 years.

The female subjects have fewer troubles with keeping their personal and environmental hygiene on daily basis; also the care for feeding and taking their prescribed medications which is not the case for the male subjects and that is shown through high rated statistical value. Furthermore the subjects who are in marriage or the ones who don't have family based obligations have also significantly less troubles in taking care for themselves. The ones who are with low level of education or the ones which are unemployed have significantly high obstacles for taking care for themselves. The statistical significance is high as well regarding the family support; moreover the subjects who are feeling that they do not have that kind of support have also troubles of taking care of themselves. The subjects who are not in a matrimonial community, unemployed or with weak support coming from their family have highly negative significance on their functioning regarding working and educational plan. The subjects from the both sexes and from the both adult groups have problems with the realization and ongoing contacts with their friends and relatives, the subjects who are singles have low rate in establishing personal or social contacts, which is not the case for the ones who are in matrimony. Furthermore the subjects who have lower education or they are not employed have significantly harder tasks in establishing of personal and social contacts versus the ones who are employed and possessing higher level of education. The subjects who have family obligations have significant problem regarding the personal and social relations, as well the subjects who do not have family support. This research is high noting the mean of the family support, having that the subjects whose state of minds are in the way that they don't have the family support very often are manifesting aggressive or disturbing behavior. The subjects who are in official working relation, the ones who do not have family obliged or the ones who are felling that they have the support from their families have statistically high significant positive level regarding of self care and more quality psychosocial functioning in comparison with the subjects who are not employed, who are family obliged or do not have family support. The subjects who have lower level of education have meaningful and more intense positive and negative schizophrenic simptomatologija on the PANSS scale in comparison with the ones who have higher education, as well the ones who are unemployed, have family obligations and do not have support from their families. Statistically significant stronger depressive simpromatology that was measured with the HAMD scale was noted at the subjects who are with family heredity. There is high statistically significant difference between the subjects who were treated daily in a hospital and the ones who are ambulatory cared, the patients who were treated on a daily basis in hospital do not have any hard times regarding their daily self care, are successful in completing their working, domestic or their educational tasks as well they have better functioning regarding their social and private relations.

The patients who were daily treated in hospitals in a period of 6 months have overall better psychosocial functioning measured on the PSP scale then the ones who were treated on ambulatory basis. The subjects who were daily treated in hospitals have shown statistically significant difference in the reduction of the positive and negative schizophrenic symptomatology versus the ones who were ambulatory treated. The results from the research have shown significant negative correlation between the expressiveness of depressive symptology and the scale of personal and social functioning versus the ones who are with more intense depressiveness, the ones with bigger reduction of negative schizophrenic symptology have better life quality, that can be shown through statistically significant negative correlation between the negative PANSS scale and the scale for social and personal functioning PSP. The subjects with schizophrenic disorder who were treated for 6 months on daily hospital basis have shown overall significant improvement in their life quality expressed through the reduction of the negative schizophrenic 
symptology and also through the improvement of their daily personal and psychological functioning, and it should be noted that this improvement statistically significant is more expressive than the subjects who were treated on ambulatory basis.

The research overall is pointing out the importance of psychosocial support expressed through accurate pharmaceutical and psychosocial treatment, supporting the family acceptance and support, supporting of the social/environmental acceptance and collaboration, with the purpose of once more the people with schizophrenia to be included in the everyday normal functioning that will gain better life quality for this individuals.

Keywords: not available.

Defended: January 20, 2012.

Mentor: Prof. Dr. Antoni Novotni.

Silvana Markovska-Simoska. Neurophysiologic correlations and endophenotypes in attention deficit with or without hyperactivity [PhD Thesis]. Skopje, Republic of Macedonia: Institute of medical and experimental physiology with anthropology, Faculty of Medicine, Ss Cyril and Methodius University of Skopje; 2012. Maced J Med Sci. 2013 Mar 15; 6(1):106-123.

Background: Attention deficit hyperactivity disorder (ADHD) is a clinically heterogeneous neurobehavioral disorder that is associated with tremendous financial costs, stress to families, adverse academic and occupational outcomes. According to the DSM-IV, ADHD is characterized by a varying amount of inattention, hyperactivity, and impulsivity symptoms. The ICD-10, although using a different name (Hyperkinetic disorder), lists similar criteria for this disorder. ADHD in adults is fairly common, though it often goes unrecognized. The worldwide prevalence of ADHD is approximately $3-10 \%$ in children and adolescents, whereby $>50 \%$ of them continue to display clinically significant symptoms after reaching the adulthood. In the last decade, many studies have tried to define the neural correlates of ADHD and because these results are very divergent, identifying of subtypes is needed.

Aim: The main aim of this study is the identification of electroencephalographic subtypes and assessment of neurophysiological indices of the executive functions in ADHD adults as a basis for defining the corresponding endophenotypes among ADHD population. The additional research is also done concerning the neuropsychological functioning of the same ADHD subjects. In relation to this, the assessment of brain-rate values and their topographic localization has been investigated as a concomitant aim. This multidimensional approach will bring improvement for the objective diagnosis of ADHD and consequently for appropriate and successful treatment.

Subjects and Methods: In this study few attention diagnostic tests of the Cogmed Testing Tool (CTT) and Amsterdam Neuropsychological Tasks (ANT) were applied. Both are battery of neuropsychological tests that allow systematic evaluation of information processing capacities. Measured and analyzed parameters were speed, speed variability and accuracy of performance. Furthermore, the absolute spectra power of the brain waves, ERPs and brainrate values were examined through quantitative EEG (QEEG) in two resting conditions (eyes closed and eyes opened) and two neuropsychological tasks (visual and emotional continuous performance tests) in 67 adults diagnosed with ADHD and 50 healthy controls.

Results: The ADHD group showed a greater response time and larger variability of reaction time than the control group, but there was not a significant difference in error types. It was also been shown that differences in performance quality increase with task complication and duration. Accordingly, the ADHD group showed more difficulties of dealing with complexity tasks.

Four distinct QEEG subtypes of adults with ADHD were selected according to the Kropotov's classification and then were attested using statistical analysis. The obtained results confirmed that the absolute spectra power of all frequency bands depends on the group qualification with the highest values of: a) slow waves (delta-theta) in the first QEEG subtype; b) theta (frontal midline) in the second; c) frontal beta in the third and d) excess alpha in the fourth one.

ERP results point out that there is disturbance in executive functioning in investigated ADHD group and in addition, the dysfunction of activation (P3bP), motor inhibition (P3supF) and monitoring (P4monCC) components is confirmed.

As expected, lower values of brain-rate were found for the first and second subtype, and higher for the third and fourth subtype.

Conclusion: From the obtained results, it can be concluded that the applied neuropsychological and neurophysiological measures relatively clearly differentiate the ADHD into four subtypes, illustrating the heterogeneous and multifactorial character of this disorder with different clinical expression, related to different underlying neuropsychological and electrophysiological abnormalities, and consequently the different responses to treatment regimes.

This study aims to advance and facilitate the pace of using combining neuropsychological and neurophysiologic procedures in clinical practice as objective measures of ADHD for better assessment, subtyping and treatment of ADHD. 
Keywords: not available.

Defended: March 30, 2012.

Mentor: Prof. Dr. Nada Pop-Jordanova.

Daniela Georgieva. Evaluation of the results of the operative procedures according to the methods of Mitchell and Keller for correction of the Hallux valgus deformity [PhD Thesis]. Skopje, Republic of Macedonia: University Clinic for Orthopedic surgery, Faculty of Medicine, Ss Cyril and Methodius University of Skopje; 2012. Maced J Med Sci. 2013 Mar 15; 6(1):106-123.

Introduction: Hallux valgus is the most frequent and important deformity of the foot, and it is characterized with a valgus position of the big toe, enlarged I intermetatarsal angle, appearance of pseudoegsostosis or bursitis in the area of the medial side of the head of the I metatarsal bone, as well as with an inside rotation of the big toe in more severe cases. This deformity is met in all nations and races, but somewhat more often in the civilized nations. Most often it can be found in the urban population, in the fourth decade of their lives, and more often in women than man. The ratio is $3: 1$. The first thorough description of hallux valgus deformity was published in 1871 by Carl Hueter. Since then the importance of the surgical correction of this deformity has been recognized. More than 100 surgical procedures have been described for the correction of this deformity. This study deals with the patients having Hallux Valgus deformity, using the clinical and radiographic analyses pre and post-operatively, according to the surgical treatment by the methods of Mitchell and Keller. The essence of these surgical procedures is to correct the deformity, to make an effort to eliminate the anatomic potential for the deformity origination, as well as to create a normal position, direction and physiologically antagonistic function of the muscles which are not balanced.

Aim: The aim of this study is to make a pre- and postoperative analysis of the treated cases by using two different surgical procedures for the correction of Hallux valgus deformity, and the clinical and radiographic results.

Material and methods: The study was worked out at the University Clinic for Orthopedic Surgery, the Faculty of Medicine in Skopje. The material for the investigation comprised 70 patients having Hallux valgus deformity. The patients were divided in two groups of 35 patients each. They were treated by the operative procedures according to the methods of Mitchell and Keller. The patients were grouped according their sex, age (in 2 groups: from 20 to 50 and over 50 years of age), and the degree of deformity (serious or severe degree of deformity). The operative procedures in both groups were performed in regional spinal anesthesia. The operative technique according to Mitchell consist double step-cut osteotomy of the distal portion of the first metatarsal bone, lateral displacement and angulation of the capital fragment and medial capsulorrhaphy. The operative treatment according to Keller is represented by decompression of the metatarsophalangeal joint by resection of one third to one half of the proximal phalanx, thereby relaxing the contacted lateral structures. Concerning the clinical methods, all symptoms were evaluated, but pain and the metatarsalgia were the most dominant appears in this deformity. Radiographic examination was done by the method of Hardy and Clapham. Evaluation of the first metatarsofalangeal joint condition was done according to Piggott classification. Besides radiopodometrical methods of the radiographies, an evaluation of the secondary arthritic and reactive changes of the first metatarsophalangeal joint was done. The clinical and radiographic examinations were done pre-operatively and post-operatively.

Results: According to the sex, the first group was represented by $3(8.57 \%)$ male patients and 32 $(91.43 \%)$ female patients, while the second group was represented by $2(5.71 \%)$ males and $33(94.29 \%)$ female patients. The average age of the patients in group 1 was $41.94 \pm 16.5$ years, while for group 2 it was $56.29 \pm 12.5$ years. The difference in the mean age between the two groups is statistically high significant $(p<0.001)$. Majority of the patients from both groups complained on pain in the area of medial eminence, namely there was pain in $33(94.3 \%)$ patients operated by the method of Mitchell, and in 34 $(97.1 \%)$ patients operated by the method of Keller. Having done the operative intervention by the method of Mitchell, pain in the area of the front foot was felt by $3(8.8 \%)$ patients, while the patients operated by the method of Keller did not suffer any pain after the operation. Metatarsalgia was registered in 25 (71.4\%) patients operated by the method of Mitchell, and in 27 (77.1\%) patients operated by Keller's technique before the operation. Post-operatively, metatarsalgia was registered in $11(32.3 \%)$ patients from the first group and $4(11.8 \%)$ patients from the second group. An average dimension of the I metatarsophalangeal angle of $40.31 \pm 7.6$ degrees was measured in the group of patients treated by the method of Mitchell, before the operation, and it is not significantly greater $(p>0.05)$ than the average dimension of the same angle in the group of patients treated by the method of Keller, where its value is $37.49 \pm 9.7$ degrees. The dimension of the I metatarsophalangeal angle in all patients was measured post-operatively during the control examinations, namely 3 moths and 1 year after the intervention. The average dimension of the I metatarsophalangeal angle in the patients operated by the method of Mitchell after 3 months was $16.97 \pm$ 3.8 degrees, while in the patients operated by the method of Keller this average dimension was smaller and was $15.0 \pm 1.7$ degrees. This difference tested by the $t$ test for independent samples was statistically high significant, or important $(p<0.01)$. During the second control examination, 1 year post-operatively, 
average dimension of the I metatarsophalangeal angle in the patients of the first group was $18.66 \pm 4.0$, and $15.83 \pm 1.9$ degrees in the patients of the second group. This difference was statistically high significant, or important $(p<0.001)$. The average dimension of the I intermetatarsal angle, 3 months after the operation was $10.83 \pm 2.4$ degrees (Mitchell), and it is significantly less $(p<0.001)$ than the average dimension of this angle treated by the method of Keller, which was $14.97 \pm 4.0$ degrees. This statistically significant difference $(p<0.001)$ in the average dimension of the I intermetatarsal angle between the two groups was confirmed 1 year after the operation, too. The patients operated by Mitchell's technique, according to Piggott classification, have deviation significantly more often on the l-st metatarsofalangeal joint of $51.43 \%$ as a result of their hallux valgus deformity. Significantly more often, there is a subluxation of $77.14 \%$ on the l-st metatarsofalangeal joint in the patients treated by Keller's technique.

Summary: Clinical and radiographic analyses are of special importance in order to make a final decision for the type of operative treatment. In this study, the operative technique according to Mitchell showed to be an effective procedure for hallux valgus and metatarsus primus varus in young and middle-aged patients, while the resectional arthroplasty according to Keller's method is a good procedure for correction of hallux valgus deformity in older patients having arthritic changes. It is expected that this wide study of this deformity will have positive results and will be important for the theory and orthopedic practice, especially because of the performed choice of operative procedures, which can be recommended for systematic follow-up of hallux valgus deformity.

Keywords: hallux valgus deformity; Mitchell; Keller; foot.

Defended: April 02, 2012.

Mentor: Prof. Dr. Anastasika Poposka.

\begin{abstract}
Ana Kaftandzieva. Extended-spectrum betalactamases producing Escherichia coli and Klebsiella spp: detection, prevalence and molecular characterization [PhD Thesis]. Skopje, Republic of Macedonia: Institute of Microbiology and Parasitology, Faculty of Medicine, Ss Cyril and Methodius University of Skopje; 2012. Maced J Med Sci. 2013 Mar 15; 6(1):106-123.
\end{abstract}

Since their first identification 25 years ago, ESBLproducing bacteria have become a serious clinical problem and cause of nosocomial infections. ESBL (extended spectrum beta lactamases) are enzymes which have the ability to hydrolyze penicillins, firstand second-generation cephalosporins, including oxyiminocephalosporins and monobactams (but not the cephamycins and carbapenems) and which are inhibited by $\beta$-lactamase inhibitors such as clavulanic acid. ESBLs are encoded by transferable conjugative plasmids in bacteria of Enterobacteriaceae family (majority of ESBL-producing organisms are E. coli and Klebsiella spp.). Most of the plasmids not only contain genes encoding ESBL enzymes, but also carry genes conferring resistance to several non-beta-lactam antibiotics. Hence, the most appropriate name for the ESBL-producing bacteria would be "multidrug resistant organisms". This resulted in limited treatment options and consequently, in clinical outcome. Another problem is the need of additional tests for detection of ESBL-production. These enzymes have different affinity to various substrates and because of the different levels of enzyme production; it is difficult to detect ESBL production by the routine disk diffusion techniques. Therefore, recommended therapy might not be effective, resulting in treatment failure and increased spread of these strains in the hospital environment. ESBL have spread globally and their prevalence varies among different medical institutions and geographic regions.

The aims of this investigation were: to determine the prevalence and distribution of ESBL producers among E. coli and Klebsiella spp. isolates from patients hospitalized at the University Clinics (UC) in Skopje; to determine the susceptibility of ESBL-positive isolates and to compare the susceptibility between ESBL-positive and ESBL-negative isolates to different groups of antimicrobial agents (beta-lactams and nonbeta-lactams); to determine genes (bla bla $_{\text {CTX-M, }}$, bla $a_{\mathrm{OXA}}$ and bla $_{\text {DHA }}$ ) encoding different ESBL types and to evaluate the association of MIC (minimal inhibitory concentration) of antimicrobial agents with $\beta$-lactamase gene types.

A total of 1207 consecutive non-repeat isolates of $E$. coli and $K$. pneumoniae- $K p$ (E. coli-804, Kp-403) were obtained from different clinical specimens (such as urine, tracheal aspirate, blood, wound swab, punctuate, endotracheal tube and canilla) from patients hospitalized at the UC in Skopje over a one year period (February 2008- January 2009). The isolates were identified on the basis of conventional microbiological procedures. Susceptibility was determined by standard disk diffusion method and by automated method using Vitek (for the determination of the MIC). A total of 251 isolates were selected ( $E$. coli-126 and K. pneumoniae-125). Modified double disk test and ESBL-agar were used as screening tests and ESBL-set, E-test and Vitek were used as confirmatory tests for phenotypic detection of the ESBL- production. A total of $100 \mathrm{ESBL}$ positive strains (E. coli-52 and K. pneumoniae-48) were randomly selected to detect the presence of genes using molecular method- multiplex PCR. K. pneumoniae ATCC 700603 was used as a positive control and Escherichia coli ATCC 25922 was used as a negative control for both phenotypic methods and for multiplex PCR.

The prevalence of ESBL-positive $E$. coli and $K$. pneumoniae was $15.7 \%$ and $31 \%$, respectively. The 
study showed the higher prevalence of ESBLproducers in isolates from surgical clinics compared to those from internal medicine clinics. Most of the ESBL-producers were from the Pediatric Clinic. The highest number of ESBL- positive E. coli and Klebsiella pneumoniae strains was isolated from urine specimen.

Determination of susceptibility of ESBL-positive isolates has shown: a) combination of betalactam/beta-lactamase inhibitor, ESBL-positive isolates of $E$. coli and $K$. pneumoniae were more susceptible to piperacillin/tazobactam compared to amoxicillin-clavulanic acid; b) cephalosporins, ESBLpositive $E$. coli isolates were more susceptible to ceftazidime (51\%), and ESBL-positive K. pneumoniae isolates were more susceptible to ceftriaxone (41\%). Considering cefepime, $44 \%$ of $E$. coli isolates and $66 \%$ of $K$. pneumoniae isolates were susceptible; c) imipenem, all ESBL- positive isolates were $100 \%$ susceptible; d) aminoglycosides, 94\% of ESBLpositive $E$. coli isolates and $66.4 \%$ of ESBL-positive $K$. pneumoniae isolates were more susceptible to amikacin than to gentamicin. e) quinolones, the percent of ESBL- positive isolates susceptible to ciprofloxacin was $34 \%$ for $E$. coli isolates and $56 \%$ for K. pneumoniae isolates; f) uroantiseptics, ESBLpositive $E$. coli isolates ( $83 \%)$ were more susceptible to nitrofurantoin than $K$. pneumoniae isolates (24\%). These data are important for recommending adequate treatment of infections with ESBL-producing bacteria. First-line therapy for ESBL-producing bacteria is iniipenem, as well as, piperacillin-tazobactam. Cefepime in high concentration and in combination with an aminoglycoside should be recommended as second-line therapy. Drug of choice for the urinary tract infections is nitrofurantoin, but only for ESBLpositive $E$. coli isolates. Comparing the susceptibility of ESBL-positive and ESBL-negative $E$. coli and $K$. pneumoniae isolates, ESBL-negative isolates were more susceptible to all tested antimicrobial agents.

In this investigation, $39 \%$ and $61 \%$ of $87 \mathrm{ESBL}$ typeable isolates harbour single ESBL gene and two or more genes for ESBL, respectively. TEM and SHV types of ESBL were frequently found in $E$. coli (11/17) and $K$. pneumoniae $(13 / 17)$, respectively. Bla $\mathrm{SHV}_{\mathrm{SH}}$ and bla ${ }_{\text {TEM }}$ were also co-present with other genes. Two or more genes for ESBL were present in $61 \%$ typeable isolates, blaj: $j_{\mathrm{TEM}}+$ bla $_{\mathrm{SHV}}$ being the most common combination $(16 \%)$. Eight strains of $E$. coli and 4 strains of $K$. pneumoniae harbour 3 genes for ESBL. Only 7 strains of $K$. pneumoniae $(8 \%)$ and none of $E$. coli harbour 4 genes for ESBL. Overall, bla ${ }_{\text {TEM }}$ was

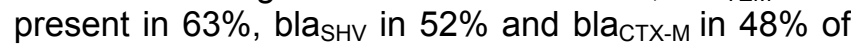
the isolates, either alone or in combinations. Considering the distribution of ESBL-genotypes in different University Clinics, the highest percent of isolates with one gene originated from the Haematology Clinic; with two genes - from Nephrology Clinic and with three genes - from Pediatric Clinic. Considering the distribution of ESBL-genotypes in different samples, the highest percent of isolates with one gene originated from blood; with two genes-from urine and with three genes- from tracheal aspirate.

In our study all ESBL-positive isolates were susceptible to imipenem, regardless of ESBL-type. Isolates harbouring 3 and more genes were characterized by the highest degree of resistance to AMC compared to those with single and two genes In a case of PIP/TAZ this difference was not statistically significant $(p>0.05)$ Isolates with $\geq 3$ genes were highly resistant to cefotaxime, ceftazidime and cefepime compared to the isolates with single or two genes. This difference was statistically significant. MICs of 64 of ceftazidime and cefotaxime were $48.3 \%$ and $79.3 \%$ of ESBL-positive isolates, respectively. Cefepime showed the best in vitro activity (except isolates with two enzyme types, such as TEM+SHV and TEM+CTX-M, to which cefepime was less active than ceftazidime). Amikacin was more active than gentamicin against all beta-lactamase types. Isolates with 3 and more beta-lactamase types showed the highest resistance to nitrofurantoin, compared to the isolates with one or two genes.

The correct identification of the genes involved in beta-lactamase mediated resistance as well as appropriate antibiotic policy, judicious usage of extended- spectrum cephalosporins, periodic surveillance of antibiotic resistance patterns and infection control measures for the surveillance are crucial to overcome the problems associated with ESBLs.

Keywords: Klebsiella spp; E. coli; $\beta$-lactamases; ESBL; multiplex PCR.

Defended: April 05, 2012.

Mentor: Prof. Dr. Nikola Panovski.

Rozeta Mileva-Peceva. Transversal analytical study about the use of food supplements on the territory of Skopje [PhD Thesis]. Skopje, Republic of Macedonia: Institute of epidemiology and biostatistics with medical informatics, Medical Faculty, Ss Cyril and Methodius University of Skopje; 2012. Maced J Med Sci. 2013 Mar 15; 6(1):106-123.

There is a trend of increasing use of vitamin and/or mineral containing food supplements (VMS) in the world. Demographic data of the users and of general practitioners that recommend these products to their patients helps create the profile of the "typical consumer" of these products and the profile of "physician that recommends" these products. Analysis of the characteristics of the product that the physician recommends, gives information and guidance for development of new products by the industry.

Objective: To determine the prevalence and demographic characteristics of the respondents for VMS use and describe the profile of "typical consumer" of these products. To determine the source 
of information that patients use for VMS products and patients views regarding VMS use. To determine the prevalence, demographic characteristics of the general practitioners that recommend VMS. To define the product's characteristics that affect physician decision to recommend the product to the patients. To determine if the availability of the personal experience or published data affect physician's decision to recommend the product. To determine the effect that marketing methods have on the physician.

Materials and Methods: The study was designed as a cross-sectional study. It was conducted in general practitioner's surgeries on the territory of Skopje. During our visit of each surgery, in the first phase we interviewed the patients and in the second phase the general practitioner. Data were collected with the method of interview using a specially designed questionnaire as an instrument of this research. We used appropriate statistical tests to analyze the data.

Results: We analyzed data on VMS use from 2000 out-patients as well as 400 general practitioners. From all interviewed individuals, 849 (42.45\%) claimed using VMS. These supplements are significantly more commonly used by women, those from Macedonian ethnicity, those that were divorced, living in the city, respondents with higher educational level and the employed ones. Patients aged over 70 years consume less VMS products compared with other age groups. Most commonly used are multivitamin products followed by vitamin $\mathrm{C}$ containing products and those with $B$ vitamins. Monocomponent supplements containing calcium and magnesium were mostly used from the mineral supplements. These products are most commonly purchased in pharmacies followed by supermarkets and the most common source of information for VMS products are the physician, magazines and television. Every other user $(58.30 \%)$ informs the physician for the use of these products.

In total, 291 (73.25\%) from the interviewed general practitioners recommend VMD to their patients in the everyday practice. Features of the general practitioner that recommends VMS to the patients are: being from Macedonian ethnicity, male gender, age below 30 years, working in suburb areas, short working experience and being a user of VMS. Characteristics from the VMS product associated with its acceptance by the physicians are: the product to be present on the market for a longer period, to have once a day dosing regime and acceptable price. Brand of the product influence the majority of the surveyed physicians to recommend that product. Brochures influence decision for recommending the product in $73.50 \%$ of the physicians, and the frequency of visits from marketing staff from the manufacturer influence $65.75 \%$ of the physicians in the study. Personal professional experience of the physician with the product, availability of published data as well as the recognition of the company manufacturer have a role in over $80 \%$ of doctors to recommend such a product to the patients.

Monitoring the use of VMS is of interest due to their contribution to total intake of vitamins and minerals in the population and as a source of information when planning public health activities.

Conclusion: This study defined the: profile of the "typical consumer" of VMS and the profile of "physician that recommends" VMS. This study contributes in understanding the factors related to VMS use in our population, on Skopje territory between $1^{\text {st }}$ February 2010 and 1 February 2011.The analysis arises the necessity to include the data on VMS use in the medical documentation of the patients in order to get deeper insight into VMS use. We defined the VMS characteristics and the methods for their marketing important for the industry for the product to be better accepted and recommended by physicians.

Keywords: epidemiological study; vitamins; minerals; use; food supplements; patients; general practitioners; Skopje.

Defended: April 06, 2012.

Mentor: Prof. Dr. Beti Zafirova-Ivanovska.

Viktor Isjanovski. Suicide and schizophrenia: Identification of the risk factors and strategy for prevention [PhD Thesis]. Skopje, Republic of Macedonia: University Psychiatry Clinic, Medical Faculty, Ss Cyril and Methodius University of Skopje; 2012. Maced J Med Sci. 2013 Mar 15; 6(1):106-123.

The suicide risk is $20 \%$ greater among people suffering from schizophrenia than among the general population. Suicide is the main reason for the morbidity and mortality in patients with schizophrenia. One out of four people with schizophrenia perform suicide, and some even during the psychiatric care. According to some authors $40 \%$ of patients have suicidal ideas, $20 \%$ to $50 \%$ of the patients with schizophrenia have suicide attempts, and $9 \%$ to $13 \%$ of them are successful. Many psychiatrists, in their suicide studies, underline the risk factors for committing a suicide among people suffering from schizophrenia and the need of preventive strategies. Suicide is the main reason for death in patients with schizophrenia, with highest risk in the middle age. History of suicidal attempts is usual in the suicidal schizophrenic sufferers, as well as the affective symptomatology and the sense of hopelessness and demoralization. The active paranoia can increase this risk; negative symptoms can reduce the risk of personally-destructible act among the schizophrenic patients. A large number of suicides happen at the time of hospitalization, or short after living the hospitals. Methodological assessment of the suicidal ideas and the aggressive treatment with 
psychological, pharmacological and social approach, are vital aspects of the management with the patients. When compared with the general population and individuals with other mental disorders, the persons suffering from schizophrenia have increased risk of mortality during the illness, accidents and violent death, especially suicide. Design on effective suicideprevention strategies depends on identification of risk factors typical for the individual or group, it strengthens the causal correlation between the risk factors and the disease and the changeability of the causable (risk) factors. It is vital that the efforts to prevent the suicide among the schizophrenic patients were designed according to demographic and clinical characteristics of this population. They were male, middle aged, single, living alone. The violent methods of deprivation of their own life's include firearms, hanging, jump from the heights and many other varieties as drowning, poisoning with drugs etc. From the clinical types of schizophrenia, the paranoid schizophrenia and not determinate schizophrenia are represented. The executors of suicides have active psychotic symptoms during the suicide, as well as depressive symptoms, and were under psychiatric treatment.

Keywords: schizophrenia; suicide attempts; risk factors.

Defended: April 11, 2012.

Mentor: Prof. Dr. Vesna Pejovska Gerazova.

Ferat Sallahu. Assessment of the prognostic factors that influence the final outcome in patients with colon obstruction due to colorectal carcinoma [PhD Thesis]. Skopje, Republic of Macedonia: University Clinic of Digestive Surgery, Medical Faculty, Ss Cyril and Methodius University of Skopje; 2012. Maced J Med Sci. 2013 Mar 15; 6(1):106-123.

Not available.

Keywords: not available.

Defended: June 14, 2012.

Mentor: Prof. Dr. Nikola Jankulovski.

Skender Zatriqi. Assessment of the value of Manhaim peritonitis index in patients with secondary and tertiary peritonitis [PhD Thesis]. Skopje, Republic of Macedonia: University Clinic of Digestive Surgery, Medical Faculty, Ss Cyril and Methodius University of Skopje; 2012. Maced J Med Sci. 2013 Mar 15; 6(1):106-123.

Not available.

Keywords: not available.

Defended: June 06, 2012.

Mentor: Prof. Dr. Vlado Janevski.
Marta Foteva. Standardisation of carpal kinematic radiographic measurements and their use in operative treatment of rheumatoid arthritis of the wrist [PhD Thesis]. Skopje, Republic of Macedonia: University Clinic for Orthopaedic Surgery, Medical Faculty, Ss Cyril and Methodius University of Skopje; 2012. Maced J Med Sci. 2013 Mar 15; 6(1):106-123.

Introduction: Many diseases of the wrist can be recognized by radiographic changes and quantified by radiographic measurements. The kinematic radiographic measurements could be the basis for kinematic studies of the wrist as well as a diagnostic, prognostic and follow- up tool for many conditions of the wrist.

Aim: This study was designed in two phases: In phase I, the aim was to identify the normal variations in the longitudinal and transverse dimensions of the wrist, and measure several indices on posteroanterior and lateral roentgenograms of the wrists of healthy population in the republic of Macedonia and by doing so, standardise the carpal radiographic kinematic parameters in normal morpho-functional relations of the wrist. In phase II, the aim was to use the same indices in a group of patients with rheumatoid arthritis of the Wrist, correlate the results with the clinical status of the wrists of the patients, determine an indication for operative treatment, postoperative follow-up- thus testing the use and significance of these kinematic radiographic measurements in a pathological sample.

Patients and Methods: In phase I- There were 100 healthy subjects (47 men and 53 women), 41 left and 59 right hands. The mean age of the subjects was 42 years $\pm 12,42$ (range 20 to 60 years). Measurements were made of the third metacarpal, the carpal height, the length of the capitate and the carpal- ulnar distance. On the AP roentgenograms, the indices, expressed as ratios, included the carpal height ratios (we used two methods-CHR by Youm and Revised $\mathrm{CHR}$ as described by Natrass) and the Carpal-Ulnar Distance Ratio. On the lateral roentgenograms, we were using the so- called "axial" method of drawing carpal axes and measuring the scapholunate (SL), capitolunate $(C L)$ and radiolunate $(R L)$ angle for assessment of malalignment. In phase II: We examined the radiographs of both hands from 30 patients ( 9 male and 21 female) with Rheumatoid Arthritis. The mean age of the patients was 47.7 years and the mean duration of the RA was 11.7 years. At the same time, we examined the clinical status of the wrists with a Clinical scoring chart. Further in our study we divided the patients in two groups. Group 1: patients who underwent dorsal arthrotenosynovectomy, and group 2: patients with no intervention.

Results: Phase I- The results for the radiographic parameters we have obtained are comparable to those we find in the literature for normal measurements and represent normal kinematic radiographic measurements for our healthy 
population. Phase II- CHR less than 0.48 was found in $83.4 \%$ of the patients, and CUDR less than 0.25 in $63.3 \%$. By correlating the radiographic measurements with the clinical status we concluded that Carpal Collapse and Ulnar Translation lead to functional limitation. There was negative correlation between the radiographic measurements and the duration of disease: the longer the duration of disease - the lower values for CHR, RCHR and CUDR. On the lateral roentgenograms we determined the presence of carpal instability in 50 hands, as follows: ulnar translocation (32 hands), VISI (14 hands), DISI (4 hands). Of these, isolated form of instability was present in 38 hands, while in large number of hands there was complex carpal instability, i.e. a combination of two patterns of instability (12 hands). The results showed improvement in the Clinical status of the patients from group 1 , with no significant changes in the radiographic measurements, while in group 2 there was no improvement, but worsening of the radiographic indices and the clinical symptoms.

Conclusion: This study shows that the described methods are reproducible and the values represent normal reference values that can be used in further kinematic studies of the wrist on our population. Also, these measurements can be used in determining the degree of kinematic dysfunction present in people with wrist abnormalities (such as rheumatoid arthritis), and in evaluation of the kinematic effectiveness of real and hypothetical treatment modalities, thus making them exceptionally valuable for the orthopaedic surgeons. Early identification of destabilizing forms of rheumatoid arthritis becomes possible, making the choice and timing of the surgical intervention easier.

Keywords: wrist; kinematics; carpal bones radiography; joint instability; arthritis, rheumatoid.

Defended: June 14, 2012.

Mentor: Not available.

Zlate Stojanoski. Infections and anti-infective strategy in hematopoietic stem cells recipients [PhD Thesis]. Skopje, Republic of Macedonia: University Clinic of Hematology, Medical Faculty, Ss Cyril and Methodius University of Skopje; 2012. Maced J Med Sci. 2013 Mar 15; 6(1):106-123.

Background: infective complications are major factor for further morbidity and mortality in stem cells recipients

Aim: We retrospectively analyzed the infective complications that occurred during an first 180 days after stem cell transplantation.

Material and methods: from September 2000 to September 2010, 163 patients (AML: 82, ALL: 9, CML:7, Hodgkin disease:24, NHL: 16, Multiple myeloma:31, SAA: 2 CLL: 1, Myelofibrosis: 1), underwent 173 (10 retransplant) cycles of high-dose chemotherapy and stem cell transplantation. 123 patients were threated with autologous and 50 with allogeneic stem cell transplantation. Male: 93 Female: 80 Median age: 36 (12-63). As a source of stem cells were used: peripherall blood in $145(90 \%)$, bone marrow: 25 (15\%), a combination peripherall blood/bone marrow: $3(5 \%)$ patients. The patients were treated in sterile room, conditioned with HEPA filters. Median duration of neutropenia (granulocyte count $<0.5 \times 10^{9} / \mathrm{L}$ ) was 12 days. The overall incidence of fever requiring antimicrobial treatment was $34 \% .12 \%$ of patients have fever unknown origin (FUO), whereas primary bacteremia occurred in $21.3 \%$, pneumonia in $9.7 \%$, severe skin infection in one patient, neutropenic enterocolitis in $9.7 \%$, acute viral B-hepatitis in 12 patient, CMV reactivation $20 \%$ in allogeneic, $0.8 \%$ in autologous recipients. The most frequently isolated were Gram-positive cocci from central venous line and sputum. Candida Albicans were predominantly isolated fungi (Candida Albicans: Non-Albieans Candida $=54 \%$ vs $46 \%$ ). Median time deferescence with antimicrobial therapy was 4 days (2-12 days). First-line therapy was successful in $66 \%$ of patients. Infection-related mortality day 180 in our study is $10 \%$ (allogeneic: $14.8 \%$ autologous: $7.5 \%$ ). Coclusions: active disease $(p=0.00078)$, initially neutropenic patients $(p=0.029)$, graft with $C D 34+$ cells $<2.0 \times 10^{6} / \mathrm{kg}$.tt. $\quad(\mathrm{p}=0.0000)$ in autologous and allogeneic recipients and GVHD and age up to 50 in allogeneic recipients $(p=0.00016)$, are major risk factors in stem cells recipients in our centre.

Keywords: not available.

Defended: June 15, 2012.

Mentor: Prof. Dr. Borche Georgievski.

Aneta Demerdzieva. Comparative analysis of the electrical brain activity in children with anorexia, anxiety and hyperactivity [PhD Thesis]. Skopje, Republic of Macedonia: University Children's Hospital, Medical Faculty, Ss Cyril and Methodius University of Skopje; 2012. Maced J Med Sci. 2013 Mar 15; 6(1):106-123.

Background: QEEG is the mathematical processing of digitally recorded EEG in order to highlight specific waveform components. Anxiety is defined as subjective sense of worry, apprehension, fear and distress. Anorexia is characterized by weight loss leading to a body weight of at least $15 \%$ below the normal. The weight loss is self-induced by avoidance of "fattening foods". Abnormalities in brain functioning in anorectic patients are very often persistent. The diagnosis of hyperkinetic disorder requires to define presence of abnormal levels of inattention and restlessness, that are pervasive across situations and persistent over time, that can be demonstrated by direct observation, and that are not caused by other disorders such as autism or affective disorders.

The aim of this study was to analyze the power spectra and spectrum weighted frequency (brain rate) 
as an indicator of general mental arousal in anxious, anorectic and hyperactive patients and to compare the results with healthy young people on the same age and gender.

Material and Methods. The diagnosis was made according to two statisistical manuals (DMS IV-R and ICD-10), medical history, neuropsychological assessment and QEEG. Results from spectra power for four conditions (eyes closed, eyes open, VCPT and ACPT) were analyzed using LORETA software and than exported to brain rate software.

Results and discussion. Calculating factorial ANOVA we found that there is strong statistical significance between results of power spectra for analyzed bands for sagittal and lateral topography between control groups of healthy subjects vs. analyzed three groups of patients. In general for anxious group the results indicated the presence of decreased delta, theta, alpha and beta activity especially in central and midline regions. In anorectic group delta waves have higher values for absolute power than control group of healthy individuals especially in central region. But, theta activity was statistically significant decreased again in central and midline region. Absolute alpha was reduced in all regions of sagital and lateral topography. Beta waves have absolute spectra power significantly lower in anorectic group vs. control group in frontal region which explains poor cognitive flexibility in this group of patients. The results indicated the presence of increased delta and theta in posterior region and decreased beta activity in all regions according sagital and lateral topography. In general decrease in all bands of the OEEG can be regarded as a specific sign of brain dysfunction. Results of QEEG assessment suggest that our patients have deviation from normal brain dynamic which might be explanation for abnormal social communication in these children. The identification of these characteristics in comparison with the $\mathrm{HBI}$ database is very simple and easy and has important implications for mean of QEEG in the assessment of children with psychophisiological problems. At the end defining specific EEG patterns is very important if we want to apply neurotherapy.

Keywords: anxiety; anorexia; hyperkinetic disorder; QEEG (quantitative electroencephalography); LORETA (low resolution electromagnetic tomografy); HBI (Human Brain Institute) database; spectrum weighted frequency $\left(\mathrm{f}_{\mathrm{b}}\right.$-brain rate).

Defended: June 21, 2012.

Mentor: Prof. Dr. Nada Pop-Jordanova.

Aspazija Sofijanova. Early detection of cerebral damage- S100B protein in biological fluids new method in neonatal medicine [PhD Thesis]. Skopje, Republic of Macedonia: University Children's Hospital, Medical Faculty, Ss Cyril and
Methodius University of Skopje; 2012. Maced J Med Sci. 2013 Mar 15; 6(1):106-123.

Not available.

Keywords: not available.

Defended: October 17, 2012.

Mentor: Prof. Dr. Katica Piperkova.

Aurora Bakali. Predictors for the forming of intracardiac thrombus in left heart in patients with systolic dysfunction and sinus ritham [PhD Thesis]. Skopje, Republic of Macedonia: University Clinic of Cardiology, Medical Faculty, Ss Cyril and Methodius University of Skopje; 2012. Maced J Med Sci. 2013 Mar 15; 6(1):106-123.

Background: Cardiac embolism accounts for more than $15 \%$ of embolic strokes. Pts with ischemic cardiomyopathy, dilated left ventricular (LV) size, lower LV ejection fraction (LVEF), and apical aneurysm seem to be at high risk for LV thrombus formation. Left atrial appendage (LAA) may be an additional source of thrombi in patients with dilated cardiomyopathy. The objectives of our study were to assess the prevalence of intracardiac left chamber thrombus and/or spontaneous echo contrast (SEC), to determine characteristics of patients with LV and LAA thrombus, to identify predictors of LV, left atrial (LA) and/or LAA thrombus and SEC presence, particularly in regard to $L V, L A$ and $L A A$ size, in sinus rhythm patients with dilated cardiomyopathy.

Patients and Methods: 101 sinus rhythm patients with dilated cardiomyopathy of mild to moderate systolic dysfunction who were not under anticoagulation therapy were included in the study, completed from November 2009 until January 2012.

Results: LV thrombus was found in $14 \%$ of our patients, while LAA thrombus in $45.5 \%$ of them. SEC was detected by TEE in $93 \%$ of patients in the LV, in $95 \%$ in LA and in $98 \%$ of cases in LAA, mostly of mild or mild to moderate degree. LVIDd correlated significantly with LA diameter $(r=0.49, p<0.01)$, LA diameter indexed for BSA $(r=0.44, p<0.01)$, LA area $(r=0.59, p<0.01)$, LA volume $(r=0.56, p<0.01)$, LA volume-indexed for BSA $(r=0.55, p<0.01)$, LAA maximal $(r=0.38, p<0.01)$ and LAA minimal area $(r=0.38, p<0.01)$, LAA emptying velocity $(r=-0.26$, $\mathrm{p}<0.01)$. LA size (diameter, indexed diameter, area, volume, indexed volume) showed a significant correlation with LAA maximal/minimal area, LAAEF and LAA emptying wave. LVESD $(\mathrm{OR}=1.03 ; 95 \% \mathrm{Cl}$ 1.055-1.319; $p=0.002)$ and transmitral peak $A$ wave $(\mathrm{OR}=\mathrm{I} .16 ; \quad 95 \% \mathrm{Cl} \quad 1.004-1.056 ; \quad p=0.02)$ were independent predictors of LV thrombus; whereas longer PTT $(\mathrm{OR}=1.23 ; 95 \% \mathrm{Cl} 1.082-1.404 ; \mathrm{p}=0.002)$, longer LV length in diastole $(\mathrm{OR}=1.83 ; 95 \% \mathrm{Cl} 1.085-$ 3.075; $p=0.02$ ) and larger LAA maximal area $(\mathrm{OR}=1.87 ; \quad 95 \% \mathrm{Cl}$ 1.334-2.614; $\mathrm{p}=0.0001)$ were independent predictors of LAA thrombus. Lower heart 
rate $(\mathrm{OR}=0.9 ; 95 \% \mathrm{Cl} 0.845-0.978 ; \mathrm{p}=0.01)$ and LVIDs $(\mathrm{OR}=1.2 ; \quad 95 \% \mathrm{Cl}$ 1.034-1.394; $\quad \mathrm{p}=0.01)$ were independent predictors of LV SEC presence; while lower LVEF (OR=0.84; 95\% Cl 0.725-0.981; $p=0.02)$ was predictor for LA SEC; and LA diameter (OR=1.46; $95 \% \mathrm{Cl} 1.001-2.146 ; p=0.05$ ) was predictor of LAA SEC.

Conclusions: The frequency of heart thrombi was high; with LA A thrombus three fold higher. We suggest that sinus rhythm patients with dilated cardiomyopathy, whose LV length during diastole is above $8.0 \mathrm{~cm}$, should be considered for TEE to exclude LAA thrombus.

Keywords: LV thrombus; LAA thrombus; TEE; dilated cardiomyopathy; sinus rhythm.

Defended: October 19, 2012.

Mentor: Prof. Dr. Ljubica Georgievska-Ismail.

Biljana Kuzmanovska. Oxygenation of the brain during the laparoscopic cholecystectomy [PhD Thesis]. Skopje, Republic of Macedonia: University Clinic for Anesthesiology, Reanimation and Intensive Care Medicine, Medical Faculty, Ss Cyril and Methodius University of Skopje; 2012. Maced J Med Sci. 2013 Mar 15; 6(1):106-123.

Creation of $\mathrm{CO}_{2}$ pneumoperitoneum during laparoscopy causes series of adverse effects. Impairment of brain oxygen saturation during laparoscopy is a result of increased intraabdominal pressure, absorbed $\mathrm{C} 02$ and increased intracranial pressure.

The aim of this study is to investigate the effect of pneumoperitoneum on brain oxygenation, the effect of duration of pneumoperitoneum on brain oxygenation, possible effects of pneumatic intermittent sequential compression (ISC) of legs on improving the oxygenation of the brain, and the correlation of the variations of the mean arterial pressure, blood oxygen saturation (pulsoxymetry), hearth rate and brain oxygenation during laparoscopy.

100 patients, ASA group 1 and 2, subjected to elective laparoscopic cholecystectomy were included in this study. The patients were divided in two groups consisted of 50 patients each, group I, control group, and group II, where ISC was applied. Oxygen saturation of the mixed venous blood from the internal jugular vein $\left(\mathrm{SjvO}_{2}\right)$ is indirect assessment of cerebral oxygen use (oxygenation of the brain). Blood samples were obtained from the bulb of the right jugular vein, as dominant side for venous drainage from the brain. Informed consent was obtained from each patient. Blood samples of $2 \mathrm{ml}$ were obtained several times during the operation; first sample immediately after the anesthesia induction in order to establish the baseline values of $\mathrm{SjvO}_{2}$, second sample immediately after the creation of pneumoperitoneum, and then, on every 15 minutes respectively until the end of the pneumoperitoneum. The last sample was obtained before the extubation of the patients. The ISC in the group II was terminated after the obtaining the last blood sample.

Blood pressure, hearth rate and puloxymetry were recorded each 10 minutes during the laparoscopy.

Results showed that average values of the oxygen saturation of the mixed venous blood from the internal jugular vein $-\mathrm{SjvO}_{2}$ levels were higher in the group II where ISC was applied $(82.3 \% \rightarrow 86.4 \% \rightarrow 85.3 \% \rightarrow$ $80.2 \% \rightarrow 82.8 \% \rightarrow 80.4 \%$ ), compared to group I, without ISC $\quad(85.5 \% \rightarrow 77.8 \% \rightarrow 80.6 \quad \% \rightarrow 83.8$ $\% \rightarrow 84.8 \%)$, statistically significant in the second and third measurement for $p<0.05$ for the second and the third measurement, e.g. after the creation of pneumoperitoneum, when the decrease in the brain oxygenation is most dramatic in the group without ISC.

The results showed that the saturation of the blood (pulsoxymetry) has statistically significant influence on the oxygenation of the brain $\left(\mathrm{SyjO}_{2}\right)$ with the coefficient of regresional analysis of 0.461 , and $p=$ 0.000000 .

The variations of the blood pressure and hearth rate have no influence on the brain oxygenation during laparoscopy with stable hemodynamic.

In conclusion, laparoscopy causes decreasing of the brain oxygenation that persists even after the termination of the pneumoperitoneum. Application of intermittent sequential compression of the legs is a simple and safe technique for preserving the brain oxygenation during laparoscopy by restoring the blood return from the legs. During laparoscopy, when the hemodynamic is stable, oxygenation of the brain depends on the oxygenation of the blood.

Keywords: laparoscopy; brain oxygenation; intermittent sequential compression.

Defended: October 19, 2012.

Mentor: Prof. Dr. Trajanka Trajkovska.

Andrijan Kartalov. Impact of subanesthetic doses of Ketamine on postoperative analgesia and plasma levels of proinflammatory cytokines (TNF $\alpha$, IL1 $\beta$, IL6) in patients with chronic cholecystitis [PhD Thesis]. Skopje, Republic of Macedonia: University Clinic for Anesthesiology, Reanimation and Intensive Care Medicine, Medical Faculty, Ss Cyril and Methodius University of Skopje; 2012. Maced J Med Sci. 2013 Mar 15; 6(1):106-123.

Introduction: Surgery and anesthesia elicit a characteristic response involving metabolism, synthesis and release of acute-phase proteins such as C- reactive protein (CRP), an increase in circulating concentrations of stress hormones and various mediators, e.g. cytokines. The inflammatory 
cytokines, such as tumor necrosis factor (TNF $\alpha$ ), interleukin-1 (IL-1 $\beta$ ) and interleukin-6 (IL-6) are all considered to be important mediators of the pathophysiological changes associated with surgery. Ketamine is acting as an $\mathrm{N}$-methyl D-aspirate receptor antagonist. Ketamine can control the perioperative pain.

The present study was designed to assess the postoperative pain and the immunomodulatory effect of sub-anesthetic dose of ketamine on the serum production of tumor necrosis factor (TNF $\alpha$ ), interleukin-1 (IL-1 $\beta$ ) and interleukin-6 (IL-6) in patients undergoing laparoscopic cholecystectomy.

Methods: A hundred patients, ASA grade I or II, undergoing elective surgical and laparoscopic cholecystectomy were recruited at the University Clinic for Anesthesiology, Reanimation and Intensive Care Medicine. Anesthesia was induced by i.v. administration of fentanyl 2-3 $\mu \mathrm{g} / \mathrm{kg}^{-1}$, propofol 1-2 $\mathrm{mg} / \mathrm{kg}^{-1}$, atracurium $0.5 \mathrm{mg} / \mathrm{kg}^{-1}$, and maintained with nitrous oxide mixtures with oxygen and sevofluorane. The patients were randomized in four equal groups. Group N1 - (laparoscopic group + K) comprised 25 patients who after 5 minutes of the induction of anesthesia received racemic ketamine $0.25 \mathrm{mg} / \mathrm{kg}^{-1}$. Group N2 - (laparoscopic group) consisted of 25 patients who received general anesthesia without ketamine. Group N3 - (surgical group + K) comprised 25 patients who after 5 minutes of the induction of anesthesia received racemic ketamine $0.25 \mathrm{mg} / \mathrm{kg}^{-1}$. Group N4 - (surgical group) consisted of 25 patients who received general anesthesia without ketamine.

Postoperatively, the patients were interviewed about the severity of pain according to visual scale (VAS) at the period of $30 \mathrm{~min}, 1,2,4,8,18,24$ and 48 hours.

TNF $\alpha, I L-1 \beta$ and IL- 6 were evaluated before surgery at 4,18 and $24 \mathrm{~h}$ after the operation with enzymelinked immunosorbent assays (ELISA) (GE Healthcare, Amersham ${ }^{\mathrm{TM}}$ ).

Statistical analysis was performed with Statistical Package of the Social Sciences (SPSS) for Windows. All values were shown as mean \pm (SD). For parametric data (serum concentration of the TNFa, IL$1 \beta$ and IL-6) groups were compared with analysis of variance (ANOVA) and Student $t$ - test for dependent and independent samples. For attributive date we used Friedman ANOVA and Mann - Whitney U Test. $A$ value of $P<0.05$ was regarded as significant.

Results: There were absence of significant statistical differences in gender, age, ASA classification and weight of participants in N1 and N2 and the N3 and N4 group.

Differences of mean values of TNFa and IL-1 $\beta$ between $\mathrm{N} 1$ and $\mathrm{N} 2$ groups in the postoperative period were not significant. Mean values of IL- 6 in the investigated group N1 were significantly lower than the mean values of IL- 6 in the investigated group N2 after the $4^{\text {th }}$ hour $(p=0.00990)$, after the $18^{\text {th }}$ hour $(p=$
0.00133 ) as well as after the $24^{\text {th }}$ hour since the surgery $(p=0.00860)$.

Difference of pain intensity according to the VAS scale has also been statistically significantly smaller in group $\mathrm{N} 1$ after $30 \mathrm{~min}, 1,2,8$ and 12 hours after surgery compared with $\mathrm{N} 2$ group. Difference of pain intensity according to the VAS scale has also been statistically significantly smaller in group N3 after 30 min, 1,4 , and 18 hours after surgery compared with N4 group.

Participants from N1 group asked the first postoperative analgesia after 82 +/- 20 minutes compared with patients in $\mathrm{N} 2$ group where this time was $48+/-14$ ( $p=0,000001)$ Participants from N3 group asked the first postoperative analgesia after 37 +/- 6 minutes compared with patients in N4 group where this time was $24+/-8(p=0,000001)$.

Discussion: Inflammatory response is associated with the release of a number of proinflammatory cytokines and acute-phase protein. TNFa, IL-1 $\beta$ and IL- 6 are major mediators of the acute-phase response in humans. IL-6 is released to plasma in the early postoperative period. It has suggested that the magnitude of the damage of tissues may related to the ability of macrophages, monocytes and fibroblasts to release IL-6, but not TNF $\alpha$ and IL-1 $\beta$.

IL-6 is produced by lymphoid and nonlymphoid cells and affects regulation of $T$ and $B$ cells, IG secretion, acute phase inflammatory reactions and hematopoiesis. TNF- $\alpha$ is an enhancer of IL-6 secretion and is produced primarily by activated monocytes and macrophages.

Anesthetic methods may affect the cytokine response to surgery changing nervous and hormone pathways. Increasing the dose of opioids during general anesthesia had a little effect on the IL- 6 response. Combining regional with general anesthesia suppressed the endocrine and metabolic response but had little influence on IL-6 increases (19). Although it is not known by what mechanism ketamine suppresses the IL-6 leaves.

Some studies report the recent discovery of the $\mathrm{N}$ Methyl-D-Aspartate (NMDA) receptor which seems to play a role in pain transmission and according to other studies, ketamine binds to these receptors with a nonselective antagonism, reducing wound hyperalgesia. The analgesic efficacy of the ketamine is linked to activation of NMDA receptors of the dorsal horn of the spinal cord. NMDA receptors at the spinal level prolong and amplify the nociceptive response (wind-up phenomenon). Ketamine acts on nicotinic and muscarinic receptors; it blocks sodium channels in the peripheral and central nervous system and interacts with opioid $p, \sigma$ and $\kappa$ receptors. Ketamine also acts as a non-competitive antagonist at the phencyclidine receptor site in the NMDA receptor complex canal. This system is involved in the 
development and maintenance of secondary hyperalgesia after tissue injury.

The preemptive analgesic effect is already seen in other abdominal surgical procedures, anterior cruciate ligament repair, cardiac surgery and abdominal hysterectomy.

Preemptive low-dose ketamine is able to produce adequate postoperative analgesia and increase the analgesic effect of tramadol. Ketamine is the only NMDA antagonist approved by Food and Drug Administration.

Some investigators have suggested that i.v. application of ketamine might have some advantages over epidural ketamine when administered with morphine in blocking nociceptive sensitization.

Conclusion: In patients undergoing elective laparoscopic cholecystectomy addition of small-dose of ketamine $\left(0.25 \mathrm{mg} / \mathrm{kg}^{-1}\right)$ after induction of anesthesia resulted in attenuation of the secretion of proinflammatory citokine IL-6 during the first postoperative day. Preemptive use of ketamine as a potent noncompetitive NMDA antagonist can improve the postoperative analgesia in the early postoperative period in these patients.

\section{Keywords: Ketamine; Postoperative pain Proinflammatory cytokines.}

Defended: October 22, 2012.

Mentor: Prof. Dr. Jordan Nojkov.

\section{Marica Pavkovikj. Analysis of the polymorphisms, Fc gamma receptor genes and human trombocitic antigens in patients with immune thrombocytopenia [PhD Thesis]. Skopje, Republic of Macedonia: University Clinic for Hematology, Medical Faculty, Ss Cyril and Methodius University of Skopje; 2012. Maced J Med Sci. 2013 Mar 15; 6(1):106-123.}

This thesis encompasses several studies aimed at providing additional insights in the pathogenesis of the most common autoimmune blood disorders: immune thrombocytopenia (ITP). In particular, we investigated three cytokine genes polymorphisms (TNFB+252A/G, TNFA-308A/G and IL1B-511T/C); two polymorphisms in Fc gamma receptor gene polymorphisms (FCGR2A-131H/R and FCGR3A-158F/V) an polymorphisms in human platelet antigens (HPA-1, -2 , -3 , and - 5) in patients with primary immune thrombocytopenia.

In this study we have analyzed three cytokine genes polymorphisms (TNFB+252A/G, TNFA-308A/G, IL1B$511 \mathrm{~T} / \mathrm{C})$ in 125 patients with primary ITP and 120 control subjects. Cytokines play important role in regulation of immune response. Cytokine genes are polymorphic, as many other genes in human genome, and some of these polymorphism influence cytokine level and function. These cytokine genes polymorphisms and their role in many autoimmune diseases were investigated in many studies in the past few decades. Our results demonstrated significantly different genotype distribution and allele frequencies for TNFB+252G/A between ITP patients and controls, $p=0.005$ and $p=0.009$. We did not found significant differences in the genotype distributions or allele frequencies for the two other genes (TNFA$308 \mathrm{~A} / \mathrm{G}$ and IL1B-511T/C). There was significantly different genotype distributions and allele frequencies for TNFA-308G/A between patients with unresponsive and responsive ITP, $p=0.016$ and $p=0.009$. We did not found significant differences in genotype distributions and allele frequencies for ILB- $511 \mathrm{C} / \mathrm{T}$ and TNFB+252G/A polymorphisms between these two groups of patients. There was no significant difference in genotype distributions and allele frequencies for all three polymorphisms between splenectomized and unsplenectomized ITP patients. The obtained data indicate that the A allele of TNFB+252G/A is more frequent in patients than in controls and that this polymorphism may play role in disease susceptibility. The A allele of TNFA-308G/A was more frequent in patients with unresponsive ITP, indicating that this gene polymorphisms may contribute to therapy resistance.

Fc receptors are members of immunoglobulin superfamily of molecules. They are found on many different cells (neutrophils, macrophages, lymphocytes, platelets) and form a critical link between the humoral and cellular immune responses. Their role is crucial in antibody mediated phagocytosis and antibody mediated cytotoxicities. Foreign microorganisms or human cells opsonised with antibodies are recognized by Fc gamma receptors on the effectors cells. Antibody mediated phagocytosis is the most important mechanism of platelet destructions in patients with ITP. Polymorphisms in Fc receptor genes influence their affinity and they may have role in many autoimmune diseases. These polymorphisms and their role in autoimmune disorders were investigated in numerous studies in the past two decades. Analysis of FCGR3A- 158F/Y polymorphisms in our study have shown significantly higher frequencies of high affinity $V$ allele in patients with ITP comparing with control subjects ( $\mathrm{V}$ allele $47.2 \%$ versus $37.5 \%$ ), $p=0.037$. We did not found significant differences in the genotype distribution or allele frequencies for FCGR2A-131H/R between patients with ITP and controls, $p=0.652$ and $p=0.478$. We have also found significantly different genotype distribution and allele frequencies for FCGR3A between patients with unresponsive and responsive ITP, $p=0.036$ and $p=0.008$ respectively. The reason for these was the higher frequency of $\mathrm{V}$ allele $(59.5 \%)$ in patients with unresponsive, comparing to $41 \%$ in patients with responsive ITP. There was no significant difference in genotype distribution and allele frequencies for FCGR2A between these two groups of patients. We did not found significant differences in genotype and allele frequencies for both gene 
polymorphisms between splenectomized and unsplenectomized ITP patients.

Knowing that the combination of different polymorphic variants may have a synergistic effect, we compared patients that had the combination of at least one high affinity allele variants (FCGR2A-131H or FCGR3A$158 \mathrm{~V}$ ) with control subjects. The combination of FCGR2A-131H and FCGR3A-158V allele was more common in patients with ITP than in control subjects ( $55 \%$ versus $40 \% ; p=0.024)$. At the same time, the combination of low affinity alleles FCGR2-131R and FCGR3A-158F was less common in. ITP patients than in control subjects $(50.4 \%$ versus $70 \% ; p=0.027)$.

Human platelet antigen (HPA) systems HPA-1, HPA2, HPA-3 and HPA-5 are components of platelet GP complexes GPIIb/lla, GPIb/IX and GPla/lla. It was shown that platelet membrane glycoprotein (GP) complexes were the main targets (autoantigens) for the autoantibodies in patients with ITP. HPA system consist of more than 12 bi-allelic antigen polymorphisms in which a base-pair substitution leads to change in an amino acid sequence of a membrane glycoprotein expressed on the platelet surface. Due to these polymorphisms, human platelet membrane glycoproteins can be recognized as alio or autoantigens and can cause different clinical conditions such as post-transfusion refractoriness to platelets, post-transfusion thrombocytopenic purpura and fetomaternal alloimmune thrombocytopenia. Allele and genotype frequencies of HPA-1, HPA-3, and HPA-5 were not significantly different between patients and healthy participants. Significant difference in allele frequency between patients and control subjects was found only for HPA-2b, $p=0.015$. These results suggests that HPA-2b allele was more frequent in patients with ITP than in controls $(0.25$ versus 0.148 ) and may be involved in the formation of specific autoepitope.

Keywords: not available.

Defended: October 24, 2012.

Mentor: Prof. Dr. Aleksandar Stojanovikj.

Kadri Hadzihamza. Violence and abuse of children and adolescents and the consequences on their mental health [PhD Thesis]. Skopje, Republic of Macedonia: University Psychiatry Clinic, Medical Faculty, Ss Cyril and Methodius University of Skopje; 2012. Maced J Med Sci. 2013 Mar 15; 6(1):106-123.

Different types of violence against adolescents are present and significant risk factor for the occurrence of different risk behaviors in the time immediately after the occurrence of violence and cause repeated to raise the risk of developing various diseases later in life of abused adolescents.

Cross section type of study was conducted on a group of secondary school students (adolescents) of the fourth year of secondary school, age ranging 18-19 years. Actual group of subjects consisted of 972 person: 537 boys and 435 girls. Structure of actual group by sex, nationality and socioeconomic status is approximately similar to the structure of the general population. Gathering of necessary data was accomplished with Adverse Childhood Experience questionnaire (in which questionnaire for stratification by sex, social structure, education, place of living e.g. city-village was incorporated) and Trauma Symptom Checklist for Children questionnaire.

Violence is present in the family circle and in the environment of adolescents: at school, on the street, at various gatherings. Patterns of violent behavior that adolescent adopt in the family circle are reproduced in their environment but there are indications that violence subjection affects neurobiological development of the adolescent brain.

Different types of violence are recognized differently by male and female adolescents: male adolescents statistically highly significantly more likely than females were exposed to psychological and sexual violence same as different types of neglect are more frequently reported by female adolescents. Different types of violence are represented differently in different ethnic groups. Socioeconomic status (especially low one) affects the occurrence of certain types of violence and neglect among adolescents. Psychological abuse is significantly more often associated with higher socioeconomic status.

There is a high degree of correlation between subjection to various types of violence (especially physical, psychological and sexual) and the occurrence of different negative effects on the mental health of adolescents (especially depression dissociation and sexual concerns). Most ACE score (concomitant subjection to many types of abuse and / or neglect) often mean severe consequences for the mental health of adolescents.

Prevention should be the first choice for long-term planning to address the problems of this nature. Preventive measures and interventions should address all levels: family, environment and society as a whole.

Keywords: not available.

Defended: October 29, 2012.

Mentor: Prof. Dr. Marija Raleva.

Tosho Plasheski. Genetic reasons for male infertility [PhD Thesis]. Skopje, Republic of Macedonia: University Clinic of Endocrinology, Diabetes and Metabolic Diseases, Medical Faculty, Ss Cyril and Methodius University of Skopje; 2012. Maced J Med Sci. 2013 Mar 15; 6(1):106-123.

The aims of this study were to determine the prevalence of the most common genetic causes of 
male infertility, such as $\mathrm{Y}$ chromosome microdeletions. sex chromosome aneuploidies and mutations in the cystic fibrosis gene, as well as to investigate the role of some potential risk factors associated with male infertility, such as partial AZFc deletions and duplications and to study the potential association of different polymorphisms with defective spermatogenesis and male infertility.

A total of 265 infertile/subfertile men were included in the study. According to the sperm counts they were classified into four groups: patients with azoospermia, severe oligo(astheno-terato)zoospermia $\left(<5 \times 10^{6} / \mathrm{ml}\right)$, mild oligo(astheno-terato)zoospermia $\left(>5 \times 10^{6} / \mathrm{ml}\right)$ and men with normo(astheno- terato)zoosoermia. The control group consisted of 250 DNA samples from fertile men, proven fathers with DNA analysis.

Eleven $Y$ chromosome microdeletions have been detected, of which eight AZFc deletions, one AZFb deletion as well as two large deletions, involving two $(A Z F b+c)$ and three $A Z F$ regions $(A Z F a+b+c)$. The prevalence of $\mathrm{Y}$ microdeletions was estimated at $3.4 \%$. The determination of the approximate size and borders of the deletions showed that six of the eight AZFC deletions are identical, with a size of $3.5 \mathrm{Mb}$. These deletions are result of a homologous recombination between b2 and b4 amplicons. The genotype/phenotype correlation showed that these patients have different sperm counts; five were azoospermic and one manifested severe oligozoospermia. The histopathological results of the tesis biopsy showed SCOS in two patients with b2/b2 deletion, HSG in one and SGA in one patient.

The QF-PCR method identified 10 patients with $X X Y$ syndrome, two $X Y Y$ and two $X X$ males. This method also allows for the detection of $Y$ chromosome microdeletions, partial AZFC deletions and duplications and androgen receptor CAG repeats. A high percentage of $45 \mathrm{X} 0 / 46 \mathrm{XY}$ mosaicism was detected among infertile men with the extensive $\mathrm{AZFb}+\mathrm{c}$ and $\mathrm{AZF} \mathrm{a}+\mathrm{b}+\mathrm{c}$ deletions.

Seven CFTR mutations, six DF508 and one G542X, were detected among the infertile men. The frequency of CFTR mutations was higher in all infertile groups in comparison to the fertile controls.

Fourteen gr/gr and five b2/b3 partial AZFc deletions were found. They were present both among infertile patients and fertile control men. Thirty two b2/b4 and four b2/b3 duplications were also identified both among infertile and fertile men. The distribution of $Y$ chromosome haplogroups showed certain differences among the different studied groups. Namely, haplogroup Rib was significantly more common among men with severe oligozoospermia, and RIa among men with mild oligozoospermia in comparison to the fertile controls. Different $Y$ chromosome haplogroups were present in men with the same AZF rearrangements, with the exception of patients with b2/b3 partial deletion who all belonged to haplogroup E3bl-M78.
We have found an association of CAG repeats longer than 26, 27 and 28 repeats and mild oligozoospermia. There was no association between the CAG repeats in the POLG gene and the polymorphisms in the folate genes (MTHFR C677T, MTHFR A1298C, MTR A2756G и MTRR A66G) with the impaired spermatogenesis in our population. The investigation of nine SNPs in eight genes that were previously found in association with azoospermia and oligozoospermia showed an association of three of them (rs5911500 in WC203413, rs3088232 in BRDT and rsl 1204546 in OR2W3) with azoospermia and/or oligozoospermia among our patients, as well.

Keywords: not available.

Defended: December 28, 2012.

Mentor: Prof. Dr. Chedomir Dimitrovski.

Ljubinka Damjanovska Krstikj. Diagnostic value of the antibodies against the modified citrullinated vimentin in rheumatoid arthritis and their correlation with the activity of the disease [PhD Thesis]. Skopje, Republic of Macedonia: University Clinic of Rheumatology, Medical Faculty, Ss Cyril and Methodius University of Skopje; 2012. Maced J Med Sci. 2013 Mar 15; 6(1):106-123.

Not available.

Keywords: not available.

Defended: December 27, 2012.

Mentor: Prof. Dr. Mane Grlichkov. 